\title{
The Real and Complex Convexity
}

\author{
Abidi Jamel \\ ABSTRACT: We prove that the holomorphic differential equation \\ $\varphi^{\prime \prime}(\varphi+c)=\gamma\left(\varphi^{\prime}\right)^{2}\left(\varphi: \mathbb{C} \rightarrow \mathbb{C}\right.$ be a holomorphic function and $\left.(\gamma, c) \in \mathbb{C}^{2}\right)$ \\ plays a classical role on many problems of real and complex convexity. The \\ condition exactly $\gamma \in\left\{1, \frac{s-1}{s} / s \in \mathbb{N} \backslash\{0\}\right\}$ (independently of the constant \\ $c$ ) is of great importance in this paper. \\ On the other hand, let $n \geq 1,\left(A_{1}, A_{2}\right) \in \mathbb{C}^{2}$, and $g_{1}, g_{2}: \mathbb{C}^{n} \rightarrow \mathbb{C}$ be \\ two analytic functions. Put $u(z, w)=\left|A_{1} w-g_{1}(z)\right|^{2}+\left|A_{2} w-g_{2}(z)\right|^{2}$, \\ $v(z, w)=\left|A_{1} w-\overline{g_{1}}(z)\right|^{2}+\left|A_{2} w-\overline{g_{2}}(z)\right|^{2}$, for $(z, w) \in \mathbb{C}^{n} \times \mathbb{C}$. We \\ prove that $u$ is strictly plurisubharmonic and convex on $\mathbb{C}^{n} \times \mathbb{C}$ if and only \\ if $n=1,\left(A_{1}, A_{2}\right) \in \mathbb{C}^{2} \backslash\{0\}$ and the functions $g_{1}$ and $g_{2}$ have a classical \\ representation form described in the present paper. \\ Now $v$ is convex and strictly psh on $\mathbb{C}^{n} \times \mathbb{C}$ if and only if $\left(A_{1}, A_{2}\right) \in$ \\ $\mathbb{C}^{2} \backslash\{0\}, n \in\{1,2\}$ and $g_{1}, g_{2}$ have several representations investigated in \\ this paper.
}

